\title{
ELECTROHYDRODYNAMIC SOLITONS IN KELVIN-HELMHOLTZ FLOW: THE CASE OF A NORMAL FIELD IN THE ABSENCE OF SURFACE CHARGES*
}

\author{
BY \\ EL SAYED F. EL SHEHAWEY \\ Ain Shams U'niversity, Egrpt
}

\begin{abstract}
Nonlinear electrohydrodynamic Kelvin-Helmholtz instability conditions are investigated. A charge-free surface separating two semi-infinite dielectric streaming fluids influenced by a normal electric field is subjected to nonlinear deformations. The method of multiple-scale perturbations is used in order to obtain two nonlinear Schrödinger (NLS) equations describing the behavior of the disturbed system. The stability and instability conditions of the perturbed system are discussed analytically. One of the two NLS equations is used to obtain the electrohydrodynamic (EHD) nonlinear cutoff wave number separating stable and unstable disturbances while the other equation is used to describe analytically the necessary condition for stability and instability for the system. For unstable cases, the solution starting with a solitary wave degenerates into a finite number of EHD solitons.
\end{abstract}

1. Introduction. The study of nonlinear dispersive waves, such as the works of Lighthill [13], Karpman [11], Whitham [34], and others, has been a subject of major interest in recent years. Due to the development of the subject of surface stabilities, fluid mechanics is considerably advanced. The applications of the results to nonlinear electrohydrodynamic stability of horizontal surfaces led to modifications of the views known from the linear theory (Mohamed and El Shehawey [17, 18]). New mechanisms were introduced which were absent in the linear analysis. The nonlinear nature of the electrohydrodynamic body force did not allow these mechanisms to appear in the linear analysis of the Rayleigh-Taylor instability.

In this paper I discuss the nonlinear conditions of stability and instability of electrohydrodynamic Kelvin-Helmholtz mechanisms in the presence of a normal field in the absence of surface charges on the interface. In the absence of convective, shear, body force instabilities, and the normal electric field, the principal instability mechanism is the Kelvin-Helmholtz mechanism (cf. Chandrasekhar [1]; Chang and Russell [2]).

We are interested in studying the coupling of electric stresses and Kelvin-Helmholtz finite amplitude surface waves. In the pure hydrodynamic Kelvin-Helmholtz instability,

\footnotetext{
* Received June 5, 1984.
} 
as the amplitude increases, the subsonic case becomes much more unstable than the supersonic case according to the nonlinear theory developed by Nayfeh and Saric [21]. For the case of an inviscid gas flowing parallel to a thin viscous liquid layer they found that, for a subsonic gas, stable linear modes continue to be stable while unstable linear modes continue to be unstable but with larger growth rates. For a supersonic gas, on the other hand, they found that linear modes do not grow indefinitely but become periodic waves. These nonlinear results are qualitatively confirmed by the experiments of Gater and L'Ecuyer [7], Saric and Marshall [26] and Gold, Otis, and Schlier [8].

Drazin [5], motivated by the experiments of Thorpe [31, 32], conducted a nonlinear stability analysis of the interface of two incompressible inviscid fluids moving with uniform velocities parallel to their interface for the cases of (a) zero surface tension and (b) equal densities. He found that in both cases unstable linear modes correspond to finite amplitude periodic waves. Since Drazin predicted periodic waves in his case and Nayfeh and Saric predicted unstable waves in the subsonic case, one concludes that the stabilizing effect of the nonlinear motion depends on the density ratio and the value of the surface tension. Nayfeh and Saric [22] exhibit the transition from instability to stability as the density ratio increases to unity or the surface tension decreases to zero. They used the method of multiple scales to analyze, to third order, the nonlinear motion for an arbitrary surface tension, and for waves whose frequency, wave number, amplitude and phase are slowly varying functions of both position and time. Maslowe and Kelley [14] analyzed, to second order, finite amplitude periodic surface waves for the case of zero surface tension. Their results do not exhibit the dependence of the wave velocity on the amplitude except near the linear neutrally stable wave number.

Weissman [33] examined the classical model of Lord Kelvin [12] with allowance for small, but finite, disturbances and for spatial as well as temporal development. By using the method of multiple scales, a generalized formulation of the amplitude equation was obtained, applicable to both stable and marginally unstable regions of parameter space. Of principal concern is the neighborhood of the critical point for instability, where weakly nonlinear solutions can be found for arbitrary initial conditions. Weissman obtained corrections to the results of Nayfeh and Saric.

Kant and Malik [10] investigated the nonlinear Kelvin-Helmholtz instability of a surface stressed by a magnetic field. They demonstrated that the waves can be unstable against modulation.

The main difference between the Kelvin-Helmholtz instability and the Rayleigh-Taylor instability is the inclusion of the term $\underline{V} \cdot \nabla \underline{V}$ in the perturbation equation, which is a nonlinear term. This led Thorpe [31,32] to find in his experiments results not predicted by the linear theory of the Kelvin-Helmholtz instability and that streaming plays a dual role in the stability criterion. In the meantime, if a normal electric field is applied to a Rayleigh-Taylor surface such that there are no surface charges on the interface, one finds that nonlinear results vary considerably from those of the linear theory and that the electric field plays a dual role in the stability criterion. Moreover, a role is played by the dielectric constants. It is important to know which of the fluids has the larger dielectric constant. We aim here to couple the two cases, namely the $\mathrm{K}-\mathrm{H}$ flow with a normal field, 
to examine how these nonlinearities interact. It is known that the linear dispersion relation for the stability of a horizontal surface separating two semi-infinite dielectric fluids streaming with velocities $V^{(1)}$ and $V^{(2)}$, influenced by gravity force and stressed by a normal electric field in the absence of surface charges is

$$
\begin{aligned}
\omega_{0}=k^{\prime} \frac{V^{(1)}+\rho V^{(2)}}{1+\rho} \pm & {\left[\frac{k^{\prime 3}}{(1+\rho)}+\frac{(1-\rho) k^{\prime}}{1+\rho}\right.} \\
& \left.\quad-\frac{\rho\left(V^{(2)}-V^{(1)}\right)^{2} k^{\prime 2}}{(1+\rho)^{2}}-k^{\prime 2} \frac{E_{0}^{(2)} E_{0}^{(1)}\left(\tilde{\varepsilon}^{(2)}-\tilde{\varepsilon}^{(1)}\right)^{2}}{(1+\rho)\left(\tilde{\varepsilon}^{(1)}+\tilde{\varepsilon}^{(2)}\right)}\right]^{1 / 2},
\end{aligned}
$$

where $k^{\prime}$ is the wave number, $\rho$ is the density ratio, $\tilde{\varepsilon}^{(1)(2)}$ are the dielectric constants of the fluids and $\omega_{0}$ is the wave frequency. The nonlinear electrohydrodynamic Kelvin-Helmholtz instability was treated by Mohamed and El Shehawey $([6,20])$ for tangential field and normal field in the presence of surface charges on the interface. Interest has been raised in a nonlinear analysis that attempts to explain the above-mentioned results demonstrated by the experiments.

One of the purposes of the present paper is to raise interest in examining the stabilizing and destabilizing nature in the nonlinear discussions. A second purpose of the present paper is paid to the cutoff wave number separating stable and unstable disturbances in an attempt to explain the results of Thorpe. I shall use the method of multiple scales described by Nayfeh [24]. The method was successfully used by Hasimoto and Ono [9], Davey and Stewartson [4] and Nayfeh [25] in studying the nonlinear hydrodynamic Rayleigh--Taylor and Kelvin-Helmholtz instabilities. The method applied here is characterized by rendering uniformly valid expansions near the cutoff wave number $k_{c_{+}}$. In fact we are led to two nonlinear Schrödinger equations describing the evolution of an electrohydrodynamic wave train propagating through the interface, one of which is valid near $k_{c_{+}}$. The unstable solution starting with a solitary wave (as an initial condition) degenerates into a finite number of EHD solitons.

2. Formulation of the problem. Consider two semi-infinite dielectric inviscid fluids separated by the plane $y=0$. The fluids are assumed to be streaming with the velocities $U^{(2)}$ and $U^{(1)}$. The upper and lower densities of the fluids are $\rho^{(2)}, \rho^{(1)}$, respectively. Both of the fluids are subject to a constant electric field in the $y$-direction $\left(E_{0}^{(2)}, E_{0}^{(1)}\right.$, respectively).

We shall assume that there are no surface charges at the surface of separation in the equilibrium state, and therefore the electric displacement is continuous at the interface, i.e.,

$$
\tilde{\varepsilon}^{(1)} E_{0}^{(1)}=\tilde{\varepsilon}^{(2)} E_{0}^{(2)}
$$

In our analysis the various quantities are nondimensionalized using the characteristic length $L=\left(T / \rho^{(1)} g\right)^{1 / 2}$ and the characteristic time $(L / g)^{1 / 2}$, where $T$ is the surface tension and $g$ is the acceleration due to gravity acting in the negative $y$-direction. The superscripts (1) and (2) refer to quantities in the lower fluid and upper fluid, respectively. 
The motion is assumed to be irrotational. The velocity potential $\phi^{\prime(1)(2)}$ describing the motion can be expressed in a nondimensional form as

$$
\phi^{\prime(1)(2)}=\left(g L^{3}\right)^{1 / 2}\left[V^{(1)(2)} x+\phi^{(1)(2)}(x, y, t)\right],
$$

where $\phi^{(1)(2)}(x, y, t)$ is the perturbation potential and $V^{(1) \cdot(2)}=U^{(1) \cdot(2)}(L g)^{-1 / 2}$. The velocity potential $\phi$ satisfies the equation

$$
\begin{array}{ll}
\frac{\partial^{2} \phi^{(2)}}{\partial x^{2}}+\frac{\partial^{2} \phi^{(2)}}{\partial y^{2}}=0, & \eta<y<\infty, \\
\frac{\partial^{2} \phi^{(1)}}{\partial x^{2}}+\frac{\partial^{2} \phi^{(1)}}{\partial y^{2}}=0, & -\infty<y<\eta .
\end{array}
$$

The solutions of $\phi$ have to satisfy at large distance the conditions

$$
\begin{array}{ll}
\left|\nabla \phi^{(2)}\right| \rightarrow 0 & \text { as } y \rightarrow \infty \\
\left|\nabla \phi^{(1)}\right| \rightarrow 0 & \text { as } y \rightarrow-\infty .
\end{array}
$$

The displacement of the interface is assumed to be of the form

$$
y=\eta(x, t) .
$$

The unit normal $\vec{N}$ to the surface is then given by

$$
\vec{N}=\frac{\nabla F}{[\nabla F \cdot \nabla F]^{1 / 2}}=-\eta_{x}\left(\eta_{x}^{2}+1\right)^{-1 / 2} \vec{e}_{x}+\left(\eta_{x}^{2}+1\right)^{-1 / 2} \vec{e}_{y},
$$

where $F=0$ is the equation of the surface of separation. The condition that the interface is moving with the fluid leads to

$$
\eta_{t}+V^{(2)(1)} \eta_{x}+\phi_{x}^{(2)(1)} \eta_{x}=\phi_{y}^{(2)(1)} \text { at } y=\eta \text {. }
$$

We also assume that the quasi-static approximation is valid and we introduce the electrostatic potentials $\psi^{(1)}$ and $\psi^{(2)}$ such that

$$
\vec{E}^{(2)(1)}=E_{0}^{(2)(1)} \vec{e}_{y}-\nabla \psi^{(2)(1)} .
$$

Therefore the differential equation satisfied by $\psi^{(2)(1)}$ is Laplace's equation

$$
\frac{\partial^{2} \psi^{(2)(1)}}{\partial x^{2}}+\frac{\partial^{2} \psi^{(2)(1)}}{\partial y^{2}}=0,
$$

along with the boundary condition that the tangential component of the electric field is continuous at the interface, so that

$$
\eta_{x} \llbracket \psi_{y} \rrbracket+\llbracket \psi_{x} \rrbracket=\eta_{x} \llbracket E_{0} \rrbracket \text { at } y=\eta,
$$

where 【 \represents the jump across the interface. Since there are no surface charges at the surface $y=\eta$, the normal electric displacement is continuous at the interface. Therefore

$$
\eta_{x} \llbracket \tilde{\varepsilon} \psi_{x} \rrbracket-\llbracket \tilde{\varepsilon} \psi_{,} \rrbracket=0 \text { at } y=\eta .
$$

The normal hydrodynamical stress is balanced by the normal electric stress. The 
balance condition is then

$$
\begin{aligned}
\phi_{t}^{(1)}+ & V^{(1)} \phi_{x}^{(1)}+\frac{1}{2}\left(\phi_{x}^{(1)^{2}}+\phi_{y}^{(1)^{2}}\right)+(1-\rho) \eta \\
= & \rho\left[\phi_{t}^{(2)}+V^{(2)} \phi_{x}^{(2)}+\frac{1}{2}\left(\phi_{x}^{(2)^{2}}+\phi_{y}^{(2)^{2}}\right)\right]+\eta_{x x}\left(1+\eta_{x}^{2}\right)^{-3 / 2} \\
& -\frac{1}{2} \llbracket \tilde{\varepsilon} \psi_{x}^{2} \rrbracket-\llbracket \tilde{\varepsilon} E_{0} \psi_{y} \rrbracket+\frac{1}{2} \llbracket \tilde{\varepsilon} \psi_{y}^{2} \rrbracket+2 \eta_{x} \llbracket \tilde{\varepsilon} E_{0} \psi_{x} \rrbracket \\
& -2 \eta_{x} \llbracket \tilde{\varepsilon} \psi_{x} \psi_{y} \rrbracket+\eta_{x}^{2} \llbracket \tilde{\varepsilon} \psi_{x}^{2} \rrbracket-\eta_{x}^{2} \llbracket \tilde{\varepsilon} E_{0}^{2} \rrbracket+2 \eta_{x}^{2} \llbracket \tilde{\varepsilon} E_{0} \psi_{y} \rrbracket \\
& -\eta_{x}^{2} \llbracket \tilde{\varepsilon} \psi_{y}^{2} \rrbracket-2 \eta_{x}^{3} \llbracket \tilde{\varepsilon} E_{0} \psi_{x} \rrbracket+2 \eta_{x}^{3} \llbracket \tilde{\varepsilon} \psi_{x} \psi_{y} \rrbracket+O\left(\eta_{x}^{4}\right) \text { at } y=\eta,
\end{aligned}
$$

where $\rho=\rho^{(2)} / \rho^{(1)}$, and the electric stress tensor is given by (Woodson and Melcher [35])

$$
\pi_{i j}=\tilde{\varepsilon} E_{i} E_{j}-\frac{1}{2} \delta_{i j} \tilde{\varepsilon} E^{2} .
$$

In the present case, the tangential stress is continuous. It was shown by Melcher [15] that this condition is equivalent to condition (2.12) in the linear perturbations in the absence of surface charges. One can show that the same result applies for higher-order perturbations. Therefore the continuity of the tangential stress is mathematically equivalent to the continuity of the normal electric displacement.

According to the method of multiple scale the variables $t, x$ may be scaled in the manner

$$
T_{n}=\varepsilon^{n} t, \quad X_{n}=\varepsilon^{n} x
$$

The perturbation quantities $\eta, \phi, \psi$ can also be expanded in the form

$$
\begin{aligned}
& \eta(x, t)=\sum_{n=1}^{3} \varepsilon^{n} \eta_{n}\left(X_{0}, X_{1}, X_{2}, T_{0}, T_{1}, T_{2}\right)+0\left(\varepsilon^{4}\right), \\
& \psi^{(2)(1)}(x, y, t)=\sum_{n=1}^{3} \varepsilon^{n} \psi_{n}^{(2)(1)}\left(X_{0}, X_{1}, X_{2}, y, T_{0}, T_{1}, T_{2}\right)+O\left(\varepsilon^{4}\right), \\
& \phi^{(2)(1)}(x, y, t)=\sum_{n=1}^{3} \varepsilon^{n} \phi_{n}^{(2)(1)}\left(X_{0}, X_{1}, X_{2}, y, T_{0}, T_{1}, T_{2}\right)+O\left(\varepsilon^{4}\right) .
\end{aligned}
$$

Substituting the expansions given by equations (2.15)-(2.17) into the set of equations (2.2). (2.3), (2.8)-(2.14) and equating terms of equal power of $\varepsilon$ on both sides of the equation we obtain the following set of equations and boundary conditions to order $\varepsilon$. (The other sets of equations to order $\varepsilon^{2}$ and $\varepsilon^{3}$ and their solutions are included in Appendix I.)

To order $\varepsilon$,

$$
\begin{gathered}
\frac{\partial^{2} \psi_{1}^{(2)(1)}}{\partial X_{0}^{2}}+\frac{\partial^{2} \psi_{1}^{(2)(1)}}{\partial y^{2}}=0, \\
\frac{\partial^{2} \phi_{1}^{(2)(1)}}{\partial X_{0}^{2}}+\frac{\partial^{2} \phi_{1}^{(2)(1)}}{\partial y^{2}}=0, \\
\llbracket \frac{\partial \psi_{1}}{\partial X_{0}} \rrbracket=\frac{\partial \eta_{1}}{\partial X_{0}} \llbracket E_{0} \rrbracket \quad \text { at } y=0,
\end{gathered}
$$




$$
\begin{gathered}
\left.\llbracket \tilde{\varepsilon} \frac{\partial \psi_{1}}{\partial y}\right]=0 \quad \text { at } y=0, \\
\frac{\partial \eta_{1}}{\partial T_{0}}-\frac{\partial \phi_{1}^{(2)(1)}}{\partial y}+V^{(2)(1)} \frac{\partial \eta_{1}}{\partial X_{0}}=0 \quad \text { at } y=0, \\
\frac{\partial \phi_{1}^{(1)}}{\partial T_{0}}-\rho \frac{\partial \phi_{1}^{(2)}}{\partial T_{0}}+(1-\rho) \eta_{1}+V^{(1)} \frac{\partial \phi_{1}^{(1)}}{\partial X_{0}}-\rho V^{(2)} \frac{\partial \phi_{1}^{(2)}}{\partial X_{0}} \\
=\frac{\partial^{2} \eta_{1}}{\partial X_{0}^{2}}-\left[\tilde{\varepsilon} E_{0} \frac{\partial \psi_{1}}{\partial y}\right] \quad \text { at } y=0,
\end{gathered}
$$

with the following solution:

$$
\begin{aligned}
& \eta_{1}=D\left(X_{1}, X_{2}, T_{1}, T_{2}\right) \exp i\left(k^{\prime} X_{0}-\omega_{0} T_{0}\right) \\
& +\bar{D}\left(X_{1}, X_{2}, T_{1}, T_{2}\right) \exp \left[-i\left(k^{\prime} X_{0}-\omega_{0} T_{0}\right)\right] \text {, } \\
& \psi_{1}^{(2)}=-\frac{E_{0}^{(2)}\left(\tilde{\varepsilon}^{(2)}-\tilde{\varepsilon}^{(1)}\right)}{\tilde{\varepsilon}^{(2)}+\tilde{\varepsilon}^{(1)}}\left\{D \exp \left[i\left(k^{\prime} X_{0}-\omega_{0} T_{0}\right)-k^{\prime} y\right]\right. \\
& \left.+\bar{D} \exp \left[-i\left(k^{\prime} X_{0}-\omega_{0} T_{0}\right)-k^{\prime} y\right]\right\}, \\
& \psi_{1}^{(1)}=\frac{E_{0}^{(1)}\left(\tilde{\varepsilon}^{(2)}-\tilde{\varepsilon}^{(1)}\right)}{\tilde{\varepsilon}^{(2)}+\tilde{\varepsilon}^{(1)}}\left\{D \exp \left[i\left(k^{\prime} X_{0}-\omega_{0} T_{0}\right)+k^{\prime} y\right]\right. \\
& \left.+\bar{D} \exp \left[-i\left(k^{\prime} X_{0}-\omega_{0} T_{0}\right)+k^{\prime} y\right]\right\} \text {, } \\
& \phi_{1}^{(2)}=-i\left(V^{(2)}-\frac{\omega_{0}}{k^{\prime}}\right)\left\{D\left(X_{1}, X_{2}, T_{1}, T_{2}\right) \exp \left[i\left(k^{\prime} X_{0}-\omega_{0} T_{0}\right)-k^{\prime} y\right]\right. \\
& \left.-\bar{D} \exp \left[-i\left(k^{\prime} X_{0}-\omega_{0} T_{0}\right)-k^{\prime} y\right]\right\} \text {, } \\
& \phi_{1}^{(1)}=i\left(V^{(1)}-\frac{\omega_{0}}{k^{\prime}}\right)\left\{D\left(X_{1}, X_{2}, T_{1}, T_{2}\right) \exp \left[i\left(k^{\prime} X_{0}-\omega_{0} T_{0}\right)+k^{\prime} y\right]\right. \\
& \left.-\bar{D} \exp \left[-i\left(k^{\prime} X_{0}-\omega_{0} T_{0}\right)+k^{\prime} y\right]\right\},
\end{aligned}
$$

where $\bar{D}$ is the complex conjugate of $D\left(X_{1}, X_{2}, T_{1}, T_{2}\right), k^{\prime}$ is the wave number, $\omega_{0}$ is the frequency of the center of the wave packet, and $\omega_{0}$ satisfies the dispersion relation

$$
\begin{aligned}
\left(V^{(1)}\right. & \left.-\frac{\omega_{0}}{k^{\prime}}\right)^{2}+\rho\left(V^{(2)}-\frac{\omega_{0}}{k^{\prime}}\right)^{2} \\
& =\frac{1}{k^{\prime}}\left[1-\rho-k^{\prime} \frac{E_{0}^{(2)} E_{0}^{(1)}\left(\tilde{\varepsilon}^{(2)}-\tilde{\varepsilon}^{(1)}\right)^{2}}{\tilde{\varepsilon}^{(2)}+\tilde{\varepsilon}^{(1)}}+k^{\prime 2}\right],
\end{aligned}
$$

or

$$
\begin{aligned}
\omega_{0}= & k^{\prime} \frac{V^{(1)}+\rho V^{(2)}}{1+\rho} \\
& \pm\left[\frac{k^{\prime 3}}{(1+\rho)}+\frac{(1-\rho) k^{\prime}}{(1+\rho)}-\rho \frac{\left(V^{(2)}-V^{(1)}\right)^{2} k^{\prime 2}}{(1+\rho)^{2}}-\frac{k^{\prime 2} E_{0}^{(2)} E_{0}^{(1)}\left(\tilde{\varepsilon}^{(2)}-\tilde{\varepsilon}^{(1)}\right)^{2}}{(1+\rho)\left(\tilde{\varepsilon}^{(2)}+\tilde{\varepsilon}^{(1)}\right)}\right]^{1 / 2} .
\end{aligned}
$$


Equation (2.30) is the same as the linear dispersion relation (1.1).

The interface of the two fluids is stable or unstable according to whether $\omega_{0}$ is real or complex. Thus the flow is stable when

$$
k^{\prime 2}+k^{\prime}\left[-\frac{E_{0}^{(2)} E_{0}^{(1)}\left(\tilde{\varepsilon}^{(2)}-\tilde{\varepsilon}^{(1)}\right)^{2}}{\left(\tilde{\varepsilon}^{(2)}+\tilde{\varepsilon}^{(1)}\right)}-\frac{\rho}{(1+\rho)}\left(V^{(2)}-V^{(1)}\right)^{2}\right]+(1-\rho)>0,
$$

and neutrally stable when $k^{\prime}=0$ and when the inequality of (2.31) is replaced by an equality. Thus the interface is linearly stable or unstable depending on whether $k^{\prime}$ is larger or smaller than

$$
k_{c_{+}}=(\rho-1)^{1 / 2}\left[\sinh \theta_{E+}+\cosh \theta_{E+}\right],
$$

where

$$
\sinh \theta_{E+}=\frac{\alpha_{E+}}{2(\rho-1)^{1 / 2}},
$$

and

$$
\alpha_{E+}=\frac{E_{0}^{(2)} E_{0}^{(1)}\left(\tilde{\varepsilon}^{(2)}-\tilde{\varepsilon}^{(1)}\right)^{2}}{\tilde{\varepsilon}^{(2)}+\tilde{\varepsilon}^{(1)}}+\frac{\rho}{(1+\rho)}\left(V^{(2)}-V^{(1)}\right)^{2} .
$$

The critical wave number $k_{c+}$ is the linear electrohydrodynamic cutoff wave number separating stable from unstable disturbances. We shall be concerned with the case $k^{\prime} \geqslant k_{c+} \cdot$

3. Schrödinger equations. The second- and third-order problems, respectively (Appendix I), admit the following solvability conditions:

$$
\begin{aligned}
& {\left[2(1+\rho) \frac{\omega_{0}}{k^{\prime}}-2\left(V^{(1)}+\rho V^{(2)}\right)\right] \frac{\partial D}{\partial T_{1}}} \\
& \quad+\left[2 k^{\prime}+(1+\rho) \frac{\omega_{0}^{2}}{k^{\prime 2}}-\frac{E_{0}^{(2)} E_{0}^{(1)}\left(\tilde{\varepsilon}^{(2)}-\tilde{\varepsilon}^{(1)}\right)^{2}}{\left(\tilde{\varepsilon}^{(2)}+\tilde{\varepsilon}^{(1)}\right)}-\left(V^{(1)^{2}}+\rho V^{(2)^{2}}\right)\right] \frac{\partial D}{\partial X_{1}}=0
\end{aligned}
$$

and

$$
\begin{aligned}
i \frac{\partial D}{\partial X_{2}}\left[-E_{0}^{(1)} E_{0}^{(2)} \frac{\left(\tilde{\varepsilon}^{(2)}-\tilde{\varepsilon}^{(1)}\right)^{2}}{\left(\tilde{\varepsilon}^{(2)}+\tilde{\varepsilon}^{(1)}\right)}+2 k^{\prime}+\frac{\omega_{0}^{2}}{k^{\prime 2}}(1+\rho)-\left(V^{(1)^{2}}+\rho V^{(2)^{2}}\right)\right] \\
\quad+2 i \frac{\partial D}{\partial T_{2}}\left[\frac{\omega_{0}}{k^{\prime}}(1+\rho)-\left(V^{(1)}+\rho V^{(2)}\right)\right] \\
\quad-\frac{(1+\rho)}{k^{\prime}} \frac{\partial^{2} D}{\partial T_{1}^{2}}+\left[1-\frac{(1+\rho)}{k^{\prime 3}} \omega_{0}^{2}\right] \frac{\partial^{2} D}{\partial X_{1}^{2}}-\frac{2 \omega_{0}(1+\rho)}{k^{\prime 2}} \frac{\partial^{2} D}{\partial X_{1} \partial T_{1}} \\
=-\bar{D} D^{2} \frac{8(1+\rho)}{k^{\prime}} \theta_{E+},
\end{aligned}
$$


where

$$
\begin{aligned}
\theta_{E++}= & -\frac{k^{\prime}}{16(1+\rho)}\left[\Omega _ { E _ { + } } k ^ { \prime 2 } \left[4\left(V^{(1)}-\frac{\omega_{0}}{k^{\prime}}\right)^{2}-4 \rho\left(V^{(2)}-\frac{\omega_{0}}{k^{\prime}}\right)^{2}\right.\right. \\
& \left.+\frac{4 E_{0}^{(2)} E_{0}^{(1)}\left(\tilde{\varepsilon}^{(2)}-\tilde{\varepsilon}^{(1)}\right)}{\left(\tilde{\varepsilon}^{(2)}+\tilde{\varepsilon}^{(1)}\right)^{2}}\left(\tilde{\varepsilon}^{(1)^{2}}-10 \tilde{\varepsilon}^{(1)} \tilde{\varepsilon}^{(2)}+\tilde{\varepsilon}^{(2)^{2}}\right)\right] \\
& +4 k^{\prime 3}\left(\left(V^{(1)}-\frac{\omega_{0}}{k^{\prime}}\right)^{2}+\rho\left(V^{(2)}-\frac{\omega_{0}}{k^{\prime}}\right)^{2}\right. \\
& +8 \frac{\tilde{\varepsilon}^{(2)^{2}} E_{0}^{(2)^{2}}\left(\tilde{\varepsilon}^{(2)^{2}}-\tilde{\varepsilon}^{(1)^{2}}\right)}{\left.\left.\left(\tilde{\varepsilon}^{(1)}+\tilde{\varepsilon}^{(2)}\right)^{2}\right)-3 k^{\prime 4}\right],} \\
\Omega_{E+}= & {\left[k^{\prime 2}\left(V^{(1)}-\frac{\omega_{0}}{k^{\prime}}\right)^{2}-\rho k^{\prime 2}\left(V^{(2)}-\frac{\omega_{0}}{k^{\prime}}\right)^{2}\right.} \\
& \left.+k^{\prime 2} E_{0}^{(2)} E_{0}^{(1)} \frac{\left(\tilde{\varepsilon}^{(2)}-\tilde{\varepsilon}^{(1)}\right)^{3}}{\left(\tilde{\varepsilon}^{(2)}+\tilde{\varepsilon}^{(1)}\right)^{2}}\right] /\left(1-\rho-2 k^{\prime 2}\right) .
\end{aligned}
$$

Equations (3.1) and (3.2) represent the evolution of the two-dimensional (EHD) wave packet propagating through the interface. These two equations can be combined together with the help of Eq. (2.30) to obtain a single equation describing the wave packets. The procedure is straightforward. Differentiating Eq. (2.30) twice with respect to $k^{\prime}$, substituting into Eq. (3.1), substituting the results into Eq. (3.2) and with a little manipulation, one obtains the following equation after replacing $T_{n}, X_{n}$ by $\varepsilon^{n} t, \varepsilon^{n} x$ :

$$
\frac{\partial D}{\partial x}+\frac{\partial D}{\partial t} \frac{d k^{\prime}}{d \omega_{0}}+\frac{1}{2} i \frac{d^{2} k^{\prime}}{d \omega_{0}^{2}} \frac{\partial^{2} D}{\partial t^{2}}=8 i \varepsilon^{2} \hat{\theta}_{E+} \bar{D} D^{2}
$$

where

$$
\begin{aligned}
\hat{\theta}_{E+}= & (1+\rho) \theta_{E+}\left[2 k^{\prime 2}-k^{\prime} \frac{E_{0}^{(2)} E_{0}^{(1)}\left(\tilde{\varepsilon}^{(2)}-\tilde{\varepsilon}^{(1)}\right)^{2}}{\left(\tilde{\varepsilon}^{(2)}+\tilde{\varepsilon}^{(1)}\right)}\right. \\
& \left.+(1+\rho) \frac{\omega_{0}^{2}}{k^{\prime}}-\left(V^{(1)^{2}}+\rho V^{(2)^{2}}\right) k^{\prime}\right]^{-1} \cdot
\end{aligned}
$$

If we use the transformation

$$
\imath=x, \quad \tau=t-\frac{d k^{\prime}}{d \omega_{0}} x
$$

Eq. (3.5) becomes

$$
\frac{\partial D}{\partial \imath}+\frac{1}{2} i \frac{d^{2} k^{\prime}}{d \omega_{0}^{2}} \frac{\partial^{2} D}{\partial \tau^{2}}=8 i \varepsilon^{2} \hat{\theta}_{E+} \bar{D} D^{2},
$$

which is a nonlinear Schrödinger equation. Eq. (3.7) is valid for all wave numbers and therefore it can be used to obtain the cutoff wave number. 
Davey [3] and Nayfeh [25] suggest that if we treat the linear dispersion equation $\omega_{0}=\Omega(k)$ as $k=\Omega^{-1}\left(\omega_{0}\right)$ and reverse the differentiation (i.e., differentiate with respect to $\omega_{0}$ instead of $k^{\prime}$ in the previous derivations) we obtain an equation similar to Eq. (3.5) but having the order of the space and time derivatives reversed. Thus we obtain an equation having a second-order spatial derivative but a first-order time derivative. The resulting equation is

$$
\frac{\partial D}{\partial t}+\frac{d \omega_{0}}{d k^{\prime}} \frac{\partial D}{\partial x}-\frac{1}{2} i \frac{d^{2} \omega_{0}}{d k^{\prime 2}} \frac{\partial^{2} D}{\partial x^{2}}=\frac{4 i \varepsilon^{2}(1+\rho) \theta_{E+}}{\left[(1+\rho) \omega_{0}-k^{\prime}\left(V^{(1)}+\rho V^{(2)}\right)\right]} \bar{D} D^{2} .
$$

Using the transformation

$$
\imath^{*}=x-\frac{d \omega_{0}}{d k^{\prime}} t, \quad \tau^{*}=t,
$$

Eq. (3.8) becomes

$$
\frac{\partial D}{\partial \tau^{*}}-\frac{1}{2} i \frac{d^{2} \omega_{0}}{d k^{\prime 2}} \frac{\partial^{2} D}{\partial \imath^{* 2}}=\frac{4 i \varepsilon^{2}(1+\rho) \theta_{E+}}{\left[(1+\rho) \omega_{0}-k^{\prime}\left(V^{(1)}+\rho V^{(2)}\right)\right]} \bar{D} D^{2},
$$

which is the second nonlinear Schrödinger equation. From Eq. (2.30) we may observe that Eq. (3.9) is not valid near the cutoff wave number. Also the solution breaks down as $\Omega_{l:+} \rightarrow \infty$. It is known that (Strauss [29]) the solution of Eq. (3.9) is bounded if

$$
\frac{d^{2} \omega_{0}}{d k^{\prime 2}} \frac{\theta_{E+}}{\left[(1+\rho) \omega_{0}-k^{\prime}\left(V^{(1)}+\rho V^{(2)}\right)\right]} \leqslant 0 .
$$

Thus Eq. (3.9) can be used to study the stability of the system away from the cutoff wave number. The system is stable if

$$
\frac{d^{2} \omega_{0}}{d k^{\prime 2}} \theta_{E+} \leqslant 0
$$

The perturbed solution of Eq. (3.9) is stable if it satisfies condition (3.11). This result was demonstrated by Nayfeh [25], Hasimoto and Ono [9] and Strauss [29].

The surface deflection can be obtained from either Eq. (3.5) or Eq. (3.8). If we choose Eq. (3.7), we may assume the time-dependent solution to be

$$
D=\frac{1}{2} b_{k} e^{i s_{k} t+\text { const }}
$$

where $b_{h}$ is a constant and

$$
s_{k}=\frac{d k^{\prime} / d \omega_{0}-\left[\left(d k^{\prime} / d \omega_{0}\right)^{2}-4 \varepsilon^{2} \hat{\theta}_{E+} b_{k}^{2} \frac{d^{2} k^{\prime}}{d \omega_{0}^{2}}\right]^{1 / 2}}{d^{2} k^{\prime} / d \omega_{0}^{2}} .
$$

We may expand Eq. (3.13) for small $\varepsilon$ and substitute for $d k^{\prime} / d \omega_{0}, d^{2} k^{\prime} / d \omega_{0}^{2}$ to get

$$
s_{k}=\varepsilon^{2} b_{k}^{2} \frac{(1+\rho) \theta_{E+}}{\left[(1+\rho) \omega_{0}-k^{\prime}\left(V^{(1)}+\rho V^{(2)}\right)\right]},
$$

for values of $k^{\prime}$ away from the cutoff wave number. Putting the value of $D$ into (2.15), 
after straightforward calculations we get

$$
\begin{aligned}
& \eta(x, t)=\varepsilon b_{k} \cos \left[k^{\prime} x-\left(\omega_{0}-\varepsilon^{2} b_{k}^{2} \frac{(1+\rho) \theta_{E+}}{\left[(1+\rho) \omega_{0}-k^{\prime}\left(V^{(1)}+\rho V^{(2)}\right)\right]}\right) t\right] \\
& \quad+\frac{1}{2} \varepsilon^{2} b_{k}^{2} \Omega_{E+} \cos 2\left[k^{\prime} x-\left(\omega_{0}-\varepsilon^{2} b_{k}^{2} \frac{(1+\rho) \theta_{E+}}{\left[(1+\rho) \omega_{0}-k^{\prime}\left(V^{(1)}+\rho V^{(2)}\right)\right]}\right) t\right]+O\left(\varepsilon^{3}\right) .
\end{aligned}
$$

4. The cutoff wave number. In this section we shall obtain the cutoff wave number using Eq. (3.7). The procedure can be carried out by recalculating $s_{k}$ in Eq. (3.13) near the cutoff wave number $k_{c_{+}}$, since Eq. (3.14) is no longer valid near $k_{c_{+}}$because $d k^{\prime} / d \omega_{0}$ is small. Therefore we evaluate $d k^{\prime} / d \omega_{0}$ near $k_{c_{+}}$. In the limit

$$
\begin{aligned}
& \frac{d k^{\prime}}{d \omega_{0}} \rightarrow\left[2(1+\rho) \omega_{0}-2 k^{\prime}\left(V^{(1)}+\rho V^{(2)}\right)\right] \\
& \times\left\{2 k^{\prime 2}-\frac{k^{\prime} E_{0}^{(2)} E_{0}^{(1)}\left(\tilde{\varepsilon}^{(2)}-\tilde{\varepsilon}^{(1)}\right)^{2}}{\left(\tilde{\varepsilon}^{(2)}+\tilde{\varepsilon}^{(1)}\right)}\right. \\
& \left.+\frac{k^{\prime}}{(1+\rho)}\left(V^{(1)}+\rho V^{(2)}\right)^{2}-k^{\prime}\left(V^{(1)^{2}}+\rho V^{(2)^{2}}\right)\right\}^{-1}, \\
& \frac{d^{2} k^{\prime}}{d \omega_{0}^{2}} \rightarrow\left[2 k^{\prime 2}-\frac{k^{\prime} E_{0}^{(2)} E_{0}^{(1)}\left(\tilde{\varepsilon}^{(2)}-\tilde{\varepsilon}^{(1)}\right)^{2}}{\left(\tilde{\varepsilon}^{(2)}+\tilde{\varepsilon}^{(1)}\right)}+\frac{k^{\prime}}{(1+\rho)}\left(V^{(1)}+\rho V^{(2)}\right)^{2}\right. \\
& \left.-k^{\prime}\left(V^{(1)^{2}}+\rho V^{(2)^{2}}\right)\right]^{-1} \\
& \cdot\left\{2\left(\frac{d k^{\prime}}{d \omega_{0}}\right)\right)^{2}\left(V^{(1)^{2}}+\rho V^{(2)^{2}}+E_{0}^{(2)} E_{0}^{(1)} \frac{\left(\tilde{\varepsilon}^{(2)}-\tilde{\varepsilon}^{(1)}\right)^{2}}{\left(\tilde{\varepsilon}^{(2)}+\tilde{\varepsilon}^{(1)}\right)}-3 k^{\prime}\right) \\
& \left.-4\left(V^{(1)}+\rho V^{(2)}\right) \frac{d k^{\prime}}{d \omega_{0}} \mid+2(1+\rho)\right\}, \\
& \Omega_{\ell+} \rightarrow \frac{k^{\prime 2} a_{1}}{1-\rho-2 k^{\prime 2}}, \quad \theta_{E+} \rightarrow \frac{k^{\prime 4}}{16(1+\rho)}\left[3 k^{\prime}-4 a_{2}-\frac{4 a_{1} a_{3} k^{\prime}}{1-\rho-2 k^{\prime 2}}\right],
\end{aligned}
$$

and finally we get

$$
\begin{aligned}
s_{k} \rightarrow & \left(\omega_{0}-\frac{k^{\prime}\left(V^{(1)}+\rho V^{(2)}\right)}{1+\rho}\right) \\
- & {\left[\left(\omega_{0}-\frac{k^{\prime}\left(V^{(1)}+\rho V^{(2)}\right)}{(1+\rho)}\right)^{2}\right.} \\
& \left.-\frac{\varepsilon^{2}}{8} \frac{k^{\prime 4}}{(1+\rho)} b_{k}^{2}\left(3 k^{\prime}-4 a_{2}-\frac{4 a_{1} a_{3} k^{\prime}}{\left(1-\rho-2 k^{\prime 2}\right)}\right)\right]^{1 / 2},
\end{aligned}
$$


where

$$
\begin{aligned}
a_{1}= & \left(V^{(1)}-\frac{V^{(1)}+\rho V^{(2)}}{(1+\rho)}\right)^{2}-\rho\left(V^{(2)}-\frac{V^{(1)}+\rho V^{(2)}}{(1+\rho)}\right)^{2} \\
& +\frac{E_{0}^{(2)} E_{0}^{(1)}\left(\tilde{\varepsilon}^{(2)}-\tilde{\varepsilon}^{(1)}\right)^{3}}{\left(\tilde{\varepsilon}^{(2)}+\tilde{\varepsilon}^{(1)}\right)^{2}}, \\
a_{2}= & \left(V^{(1)}-\frac{V^{(1)}+\rho V^{(2)}}{(1+\rho)}\right)^{2}+\rho\left(V^{(2)}-\frac{V^{(1)}+\rho V^{(2)}}{(1+\rho)}\right)^{2} \\
& +8 \frac{\tilde{\varepsilon}^{(2)^{2}} E_{0}^{(2)^{2}}\left(\tilde{\varepsilon}^{(2)}-\tilde{\varepsilon}^{(1)}\right)}{\left(\tilde{\varepsilon}^{(2)}+\tilde{\varepsilon}^{(1)}\right)^{2}}, \\
a_{3}= & \left(V^{(1)}-\frac{V^{(1)}+\rho V^{(2)}}{(1+\rho)}\right)^{2}-\rho\left(V^{(2)}-\frac{V^{(1)}+\rho V^{(2)}}{(1+\rho)}\right)^{2} \\
& +\frac{E_{0}^{(2)} E_{0}^{(1)}\left(\tilde{\varepsilon}^{(2)}-\tilde{\varepsilon}^{(1)}\right)}{\left(\tilde{\varepsilon}^{(2)}+\tilde{\varepsilon}^{(1)}\right)^{2}}\left(\tilde{\varepsilon}^{(1)^{2}}-10 \tilde{\varepsilon}^{(1)} \tilde{\varepsilon}^{(2)}+\tilde{\varepsilon}^{(2)^{2}}\right) .
\end{aligned}
$$

The surface deflection is then

$$
\eta(x, t)=\varepsilon b_{k} \cos \left(k^{\prime} x-\hat{s}_{k} t\right)+\frac{1}{2} \varepsilon^{2} b_{k} \Omega_{E+} \cos 2\left(k^{\prime} x-\hat{s}_{k} t\right)+O\left(\varepsilon^{3}\right),
$$

where $\hat{s}_{k}=\omega_{0}-s_{k}$. Since the principle of overstability is valid in the linear theory, it must be taken into consideration in the higher-order perturbations. Therefore, the nonlinear cutoff wave number can be obtained by requiring the vanishing of the square root in $\hat{s}_{k}$, on substituting for $\omega_{0}$. The condition becomes

$$
\begin{aligned}
& 6 \varepsilon^{2} b_{k}^{2} k^{\prime 6}-8 \varepsilon^{2} b_{k}^{2} a_{2} k^{\prime 5}+\left[-16+\varepsilon^{2} b_{k}^{2}\left(4 a_{1} a_{3}+3(1-\rho)\right)\right] k^{\prime 4} \\
& +4 k^{\prime 3}\left[4 \alpha_{E+}+\varepsilon^{2} b_{k}^{2} a_{2}(1-\rho)\right]-8 k^{\prime 2}(1-\rho) \\
& -8 \alpha_{E+} k^{\prime}(1-\rho)+8(1-\rho)^{2}=0
\end{aligned}
$$

Assuming the nonlinear cutoff wave number to be

$$
k^{\prime}=k_{c+}+\varepsilon^{2} k_{2}+O\left(\varepsilon^{4}\right),
$$

we get from Eq. (4.7) to the zeroth order in $\varepsilon$

$$
k^{\prime}{ }_{++}=\sqrt{\rho-1}\left[\sinh \theta_{E+}+\cosh \theta_{E+}\right] \text {, }
$$

and to order $\varepsilon^{2}$

$$
k_{2}=\frac{3}{16} b_{k}^{2} k_{c+}^{3} \frac{2 k_{c+}^{3}-\frac{8}{3} a_{2} k_{c+}^{2}+\left[\frac{4}{3} a_{1} a_{3}-(1-\rho)\right] k_{c+}+\frac{4}{3} a_{2}(1-\rho)}{4 k_{c+}^{3}-3 \alpha_{k+} k_{c+}^{2}+k_{c+}(1-\rho)+\frac{1}{2} \alpha_{E+}(1-\rho)} .
$$

The increment $k_{2}$ in the linear cutoff wave number $k_{c+}$ may be positive or negative according to Eq. (4.10). The sign of $k_{2}$ depends on the applied electric field, the density ratio $\rho$ and the streaming velocities. We observe that contrary to the linear theory, the perpendicular electric field is not strictly destabilizing. This result agrees with the 
observations of Taylor [30] and Smith and Melcher [28]. Taylor observed experimentally that there are stable oscillations near the linear cutoff wave number. Smith and Melcher found in their experiments on the Rayleigh-Taylor instability that the electric field plays a dual role.

5. Stable and unstable condition of the (2NLS) equation. If we use the transformation

$$
\tau^{*}=\lambda^{-1} t, \quad \imath^{*}=\lambda^{-1}\left[x-\left(\frac{d \omega_{0}}{d k^{\prime}}\right) t\right],
$$

Eq. (3.8) becomes

$$
i \frac{\partial D}{\partial \tau^{*}}+\frac{\partial^{2} D}{\partial \imath^{* 2}}+\lambda \mu D^{2} \bar{D}=0,
$$

where

$$
\begin{gathered}
\lambda=\frac{1}{2} \frac{d^{2} \omega_{0}}{d k^{\prime 2}} \\
\mu=4 \varepsilon^{2}(1+\rho) \theta_{l:+}\left[(1+\rho) \omega_{0}-k^{\prime}\left(V^{(1)}+\rho V^{(2)}\right)\right]^{-1} .
\end{gathered}
$$

Equation (5.1) can be used to discuss the stable and unstable solutions, since it is valid provided that $k^{\prime}$ is away from $k_{c_{+}}$and $\Omega_{k_{+}+}$is finite. We observe that $\Omega_{l_{+}} \rightarrow \infty$ as $k^{\prime} \rightarrow k^{*}$ where

$$
k^{*}=\left[\frac{1}{2}(1-\rho)\right]^{1 / 2},
$$

which corresponds to the second harmonic resonance. In general, it is known that the solution of Eq. (5.1) is bounded if condition (3.11) is satisfied, namely,

$$
\lambda \mu \leqslant 0 \text {. }
$$

In the limiting case as the surface tension $(T)$ tends to zero and $k^{\prime} \rightarrow 0$, we find that $\theta_{l:}$, and $d^{2} \omega_{0} / d k^{\prime 2}$ have the same sign for all wave numbers if $\rho<1$ and $k^{\prime}>k_{c_{+}}$. Therefore gravity waves are unstable. Also, as $T \rightarrow \infty$ and $k \rightarrow \infty$,

$$
\begin{aligned}
\left.\theta_{E+}\right|_{k^{\prime} \rightarrow \infty} & \rightarrow \frac{k^{\prime 5}}{8(1+\rho)}\left[\frac{(1-\rho)^{2}}{(1+\rho)^{2}}-\frac{1}{2}\right], \\
\frac{d^{2} \omega_{0}}{d k^{\prime 2}} & \rightarrow \frac{3}{4 k^{-1 / 2}(1+\rho)^{1 / 2}} .
\end{aligned}
$$

Thus $\theta_{l:+}$ and $d^{2} \omega_{0} / d k^{\prime 2}$ have different signs and, hence, capillary waves are stable only if

$$
\left[\frac{(1-\rho)^{2}}{(1+\rho)^{2}}-\frac{1}{2}\right]<0
$$

and $k^{\prime}>k_{1}$, , that is,

$$
\rho>3-2 \sqrt{2} .
$$

We may note that neither the electric field nor the streaming velocities $V^{(1)}, V^{(2)}$ have effects on the stability of gravity waves and capillary waves in the limiting cases as $T \rightarrow 0$ and $T \rightarrow x$. 
In general the stability conditions depend on the parameters $E_{0}, \tilde{\varepsilon}^{(1)(2)}, V^{(1)(2)}, k^{\prime}, \rho$ for finite $T$.

The condition $\lambda \mu=0$ splits into two equations

$$
\begin{aligned}
& {\left[2(1+\rho) \omega_{0}-2 k^{\prime}\left(V^{(1)}+\rho V^{(2)}\right)\right]^{-2}\left[1-\rho-2 k^{\prime} E_{0}^{(2)} E_{0}^{(1)} \frac{\left(\tilde{\varepsilon}^{(2)}-\tilde{\varepsilon}^{(1)}\right)^{2}}{\left(\tilde{\varepsilon}^{(2)}+\tilde{\varepsilon}^{(1)}\right)}\right.} \\
& \left.+3 k^{\prime 2}+2 \omega_{0}\left(V^{(1)}+\rho V^{(2)}\right)-2 k^{\prime}\left(V^{(1)^{2}}+\rho V^{(2)^{2}}\right)\right]^{2} \\
& =\frac{\left(V^{(1)}+\rho V^{(2)}\right)}{(1+\rho)\left[(1+\rho) \omega_{0}-2 k^{\prime}\left(V^{(1)}+\rho V^{(2)}\right)\right]} \\
& \times\left\{(1-\rho)-2 k^{\prime} E_{0}^{(2)} E_{0}^{(1)} \frac{\left(\tilde{\varepsilon}^{(2)}-\tilde{\varepsilon}^{(1)}\right)^{2}}{\tilde{\varepsilon}^{(2)}+\tilde{\varepsilon}^{(1)}}\right. \\
& \left.+3 k^{\prime 2}+2 \omega_{0}\left(V^{(1)}+\rho V^{(2)}\right)-2 k^{\prime}\left(V^{(1)^{2}}+\rho V^{(2)^{2}}\right)\right\} \\
& +\frac{3 k^{\prime}}{(1+\rho)}-\frac{E_{0}^{(2)} E_{0}^{(1)}\left(\tilde{\varepsilon}^{(2)}-\tilde{\varepsilon}^{(1)}\right)^{2}}{\left(\tilde{\varepsilon}^{(2)}+\tilde{\varepsilon}^{(1)}\right)}-\frac{V^{(1)^{2}}+\rho V^{(2)^{2}}}{(1+\rho)}, \\
& \frac{4 k^{\prime 2}}{\left(1-\rho-2 k^{\prime 2}\right)}\left[k^{\prime 2}\left(V^{(1)}-\frac{\omega_{0}}{k^{\prime}}\right)^{2}-\rho k^{\prime 2}\left(V^{(2)}-\frac{\omega_{0}}{k^{\prime}}\right)^{2}+k^{\prime 2} E_{0}^{(2)} E_{0}^{(1)} \frac{\left(\tilde{\varepsilon}^{(2)}-\tilde{\varepsilon}^{(1)}\right)^{3}}{\left(\tilde{\varepsilon}^{(2)}+\tilde{\varepsilon}^{(1)}\right)^{2}}\right] \\
& \times\left(\left(V^{(1)}-\frac{\omega_{0}}{k^{\prime}}\right)^{2}-\rho\left(V^{(2)}-\frac{\omega_{0}}{k^{\prime}}\right)^{2}\right. \\
& \left.+\frac{E_{0}^{(2)} E_{0}^{(1)}\left(\tilde{\varepsilon}^{(2)}-\tilde{\varepsilon}^{(1)}\right)}{\left(\tilde{\varepsilon}^{(2)}+\tilde{\varepsilon}^{(1)}\right)^{2}}\left(\tilde{\varepsilon}^{(1)^{2}}-10 \tilde{\varepsilon}^{(1)} \tilde{\varepsilon}^{(2)}+\tilde{\varepsilon}^{(2)^{2}}\right)\right) \\
& =-4 k^{\prime 3}\left[\left(V^{(1)}-\frac{\omega_{0}}{k^{\prime}}\right)^{2}+\rho\left(V^{(2)}+\frac{\omega_{0}}{k^{\prime}}\right)^{2}+8 \frac{\tilde{\varepsilon}^{(2)^{2}} E_{0}^{(2)^{2}}\left(\tilde{\varepsilon}^{(2)}-\tilde{\varepsilon}^{(1)}\right)}{\left(\tilde{\varepsilon}^{(2)}+\tilde{\varepsilon}^{(1)}\right)^{2}}\right]+3 k^{\prime 4} \text {, }
\end{aligned}
$$

provided that

$$
k^{2} \neq \frac{1}{2}(1-\rho) .
$$

The case $k^{2}=(1 / 2)(1-\rho)$ represents the second harmonic resonance of the system. From Eq. (5.8) we observe that the coefficients depend on the sign of $\tilde{\varepsilon}^{(1)}-\tilde{\varepsilon}^{(2)}$. This implies that the number of positive roots of the equality of Eq. (5.8) depends on the sign of $\tilde{\varepsilon}^{(1)}-\tilde{\varepsilon}^{(2)}$. Therefore the stability criterion depends on which one of the fluids has a larger dielectric constant. This agrees with the corresponding result of the Rayleigh-Taylor instability (Mohamed and El Shehawey [17, 18]).

On the other hand if the condition (5.3) is not satisfied then the system is unstable, and solitary waves propagate through the interface. It is known that (Zakharov and Shabat 
[36]) the unstable solution of the (2NLS) equation (5.1), starting with a solitary wave (as an initial condition), degenerates into a finite number of EHD solitons. Thus a necessary condition for electrohydrodynamic solitary waves to exist is that

$$
\lambda \mu>0 \text {. }
$$

6. EHD solitons. The general solution of Eq. (5.1) (Scott et al. [27]) is

$$
D=\Phi_{1}\left[1-\left\{\left(1-\frac{\Phi_{1}^{2}}{\Phi_{2}^{2}}\right) \operatorname{sn}^{2}\left[\sqrt{\frac{\lambda \mu}{2}}\left(\imath^{*}-U_{e} \tau^{*}\right)\right]\right\}\right]^{-1 / 2} \exp \left[i \frac{U_{e}}{2}\left(\imath^{*}-U_{c} \tau^{*}\right)\right]
$$

where $\Phi_{1}$ and $\Phi_{2}$ are arbitrary functions determined by the initial conditions, $U_{e}$ is the envelope velocity, $U_{c}$ is the carrier velocity and $s n$ is the Jacobi elliptic function.

For some special cases we may obtain the following envelope soliton solutions (Scott et al. [27]):

$$
D\left(\imath^{*}, \tau^{*}\right)=\Phi_{0} \operatorname{sech}\left[\sqrt{\frac{\lambda \mu}{2}} \Phi_{0}\left(\iota^{*}-U_{e} \tau^{*}\right)\right] \exp \left[i \frac{U_{e}}{2}\left(\iota^{*}-U_{c} \tau^{*}\right)\right]
$$

For the initial conditions

$$
D\left(r^{*}, 0\right)=\operatorname{sech} r^{*},
$$

Miles [16] obtained a complete solution of the problem using the inverse scattering method technique. The solutions for the bound state are (Miles [16])

$$
D_{N}\left(\imath^{*}, \tau^{*}\right)=\left(\frac{8}{\lambda \mu}\right)^{1 / 2} \bar{A}(I+\bar{B} B)^{-1} \bar{A}
$$

where

$$
\begin{aligned}
A & \equiv\left[A_{n}\right]=\left[C_{n}^{1 / 2} \exp \left(i \xi_{n} \imath^{*}+2 i \xi_{n}^{2} \tau^{*}\right)\right], \\
B & \equiv\left[B_{m n}\right]=\left[A_{m} \bar{A}_{n}\left(\xi_{m}-\bar{\xi}_{n}\right)^{-1}\right],
\end{aligned}
$$

Here, $I$ is the identity matrix, the bar denotes the complex conjugate and

$$
C_{n}=\frac{\Gamma\left(2\left(\frac{\lambda \mu}{2}\right)^{1 / 2}-n+1\right)}{\Gamma(n) \Gamma^{2}\left(\left(\frac{\lambda \mu}{2}\right)^{1 / 2}-n+1\right)},
$$

$\xi_{n}=k_{n}+i \tilde{n}_{n}$, where $\left|\xi_{1}\right|>\left|\xi_{2}\right|>\ldots>\left|\xi_{N}\right|$ and $\xi_{n}$ 's are the zaros of the analytic continuation of a $(\xi)$ in $\operatorname{Im} \xi>0$, where

$$
a(k)=\frac{\Gamma^{2}\left(\frac{1}{2}-i k\right)}{\Gamma\left(\frac{1}{2}+\left(\frac{\lambda \mu}{2}\right)^{1 / 2}-i k\right) \Gamma\left(\frac{1}{2}-\left(\frac{\lambda \mu}{2}\right)^{1 / 2}-i k\right)} .
$$

The zeros of a $(\xi)$ in $\operatorname{Im} \xi>0$ correspond to the poles of

$$
\Gamma\left(\gamma-\left(\frac{\lambda \mu}{2}\right)^{1 / 2}\right) \text { at }\left(\frac{\lambda \mu}{2}\right)^{1 / 2}-\frac{1}{2}+i k=0,1, \ldots, N-1,
$$


where $N$ is the integral part of $\left(\frac{\lambda \mu}{2}\right)^{1 / 2}$. Replacing $k$ by $k+i \tilde{\eta}$ we get

$$
\begin{aligned}
& k_{n}=0, \quad \tilde{\eta}_{n}=\frac{1}{2}+\left(\frac{\lambda \mu}{2}\right)^{1 / 2}-n, \quad n=1,2, \ldots, N, \\
& N \leqslant\left(\frac{\lambda \mu}{2}\right)^{1 / 2}<N+1 .
\end{aligned}
$$

The result is then $N$ centered solitons all moving with the same speed, where $N$ is the integral part of $(\lambda \mu / 2)^{1 / 2}$.

We note that solitons are possible only if

$$
\lambda \mu \geqslant 2 \text {. }
$$

For $0<\lambda \mu<2$, no solitons can exist and we are left with only solitary waves. For a single soliton we have the solution

$$
\begin{aligned}
D_{1}\left(\imath^{*}, \tau^{*}\right)= & {\left[2-\left(\frac{\lambda \mu}{2}\right)^{-1 / 2}\right] \exp \left[i\left(2\left(\frac{\lambda \mu}{2}\right)^{1 / 2}-1\right)^{2} \tau^{*}\right] } \\
& \times \operatorname{sech}\left\{\left(2\left(\frac{\lambda \mu}{2}\right)^{1 / 2}-1\right) \imath^{*}+\ln \left[\frac{\Gamma^{2}\left(\left(\frac{\lambda \mu}{2}\right)^{1 / 2}\right)}{\Gamma\left(2\left(\frac{\lambda \mu}{2}\right)^{1 / 2}-1\right)}\right]\right\},
\end{aligned}
$$

where $\lambda \mu$ satisfy the condition

$$
2 \leqslant \lambda \mu<8
$$

In case of equality (i.e., $\lambda \mu=2$ ) we get the solution

$$
D_{1}\left(\imath^{*}, \tau^{*}\right)=e^{i \tau^{*}} \operatorname{sech} \imath^{*} \text {. }
$$

Appendix I. For order $\varepsilon^{2}$ we get

$$
\begin{gathered}
\frac{\partial^{2} \psi_{2}^{(2)(1)}}{\partial X_{0}^{2}}+\frac{\partial^{2} \psi_{2}^{(2)(1)}}{\partial y^{2}}=-2 \frac{\partial^{2} \psi_{1}^{(2)(1)}}{\partial X_{0} \partial X_{1}}, \\
\frac{\partial^{2} \phi_{2}^{(2)(1)}}{\partial X_{0}^{2}}+\frac{\partial^{2} \phi_{2}^{(2)(1)}}{\partial y^{2}}=-2 \frac{\partial^{2} \phi_{1}^{(2)(1)}}{\partial X_{0} \partial X_{1}}, \\
\llbracket \frac{\partial \psi_{2}}{\partial X_{0}} \rrbracket=\left[\frac{\partial \eta_{2}}{\partial X_{0}}+\frac{\partial \eta_{1}}{\partial X_{1}}\right) \llbracket E_{0} \rrbracket-\llbracket \frac{\partial \psi_{1}}{\partial X_{1}} \rrbracket-\eta_{1} \llbracket \frac{\partial^{2} \psi_{1}}{\partial y \partial X_{0}} \rrbracket-\frac{\partial \eta_{1}}{\partial X_{0}} \llbracket \frac{\partial \psi_{1}}{\partial y} \rrbracket, \\
\frac{\partial \eta_{2}}{\partial T_{0}}-\frac{\partial \phi_{2}^{(2)(1)}}{\partial y}+V^{(2)(1)} \frac{\partial \eta_{2}}{\partial X_{0}} \quad \text { at } y=0 \\
=-\frac{\partial \eta_{1}}{\partial T_{1}}+\eta_{1} \frac{\partial^{2} \phi_{1}^{(2)(1)}}{\partial y^{2}}-\frac{\partial \eta_{1}}{\partial X_{0}} \frac{\partial \phi_{1}^{(2)(1)}}{\partial X_{0}}-V^{(2)(1)} \frac{\partial \eta_{1}}{\partial X_{1}} \quad \text { at } y=0,
\end{gathered}
$$




$$
\begin{aligned}
\frac{\partial \phi_{2}^{(1)}}{\partial T_{0}} & -\rho \frac{\partial \phi_{2}^{(2)}}{\partial T_{0}}+(1-\rho) \eta_{2}-\frac{\partial^{2} \eta_{2}}{\partial X_{0}^{2}}+V^{(1)} \frac{\partial \phi_{2}^{(1)}}{\partial X_{0}}-\rho V^{(2)} \frac{\partial \phi_{2}^{(2)}}{\partial X_{0}}+\llbracket \tilde{\varepsilon} E_{0} \frac{\partial \psi_{2}}{\partial y} \rrbracket \\
& =-\eta_{1} \frac{\partial^{2} \phi_{1}^{(1)}}{\partial T_{0} \partial y}-\frac{\partial \phi_{1}^{(1)}}{\partial T_{1}}+\rho \eta_{1} \frac{\partial^{2} \phi_{1}^{(2)}}{\partial T_{0} \partial y} \\
& +\rho \frac{\partial \phi_{1}^{(2)}}{\partial T_{1}}-\frac{1}{2}\left(\frac{\partial \phi_{1}^{(1)}}{\partial X_{0}}\right)^{2}+\frac{1}{2} \rho\left(\frac{\partial \phi_{1}^{(2)}}{\partial X_{0}}\right)^{2}-\frac{1}{2}\left(\frac{\partial \phi_{1}^{(1)}}{\partial y}\right)^{2} \\
& +\frac{1}{2} \rho\left(\frac{\partial \phi_{1}^{(2)}}{\partial y}\right)^{2}-V^{(1)} \eta_{1} \frac{\partial^{2} \phi_{1}^{(1)}}{\partial y \partial X_{0}}-V^{(1)} \frac{\partial \phi_{1}^{(1)}}{\partial X_{1}}+-\rho V^{(2)} \eta_{1} \frac{\partial^{2} \phi_{1}^{(2)}}{\partial y \partial X_{0}} \\
& +\rho V^{(2)} \frac{\partial \phi_{1}^{(2)}}{\partial X_{1}}+2 \frac{\partial^{2} \eta_{1}}{\partial X_{0} \partial X_{1}}-\frac{1}{2} \llbracket \tilde{\varepsilon}\left(\frac{\partial \psi_{1}}{\partial X_{0}}\right)^{2} \rrbracket-\eta_{1} \llbracket \tilde{\varepsilon} E_{0} \frac{\partial^{2} \psi_{1}}{\partial y^{2}} \rrbracket \\
& +\frac{1}{2} \llbracket \tilde{\varepsilon}\left(\frac{\partial \psi_{1}}{\partial y}\right)^{2} \rrbracket+2 \frac{\partial \eta_{1}}{\partial X_{0}} \llbracket \tilde{\varepsilon} E_{0} \frac{\partial \psi_{1}}{\partial X_{0}} \rrbracket-\left(\frac{\partial \eta_{1}}{\partial X_{0}}\right)^{2} \llbracket \tilde{\varepsilon} E_{0}^{2} \rrbracket, \quad \text { at } y=0 .
\end{aligned}
$$

Substituting the solutions of the first-order problem into the above set of equations, we get the following solutions:

$$
\begin{aligned}
\eta_{2}= & \Omega_{E:+}\left\{D^{2} \exp \left[2 i\left(k^{\prime} X_{0}-\omega_{0} T_{0}\right)\right]+\bar{D}^{2} \exp \left[-2 i\left(k^{\prime} X_{0}-\omega_{0} T_{0}\right)\right]\right\}, \\
\psi_{2}^{(2)}= & -i E_{0}^{(2)} \frac{\tilde{\varepsilon}^{(2)}-\tilde{\varepsilon}^{(1)}}{\tilde{\varepsilon}^{(2)}+\tilde{\varepsilon}^{(1)}} y \frac{\partial D}{\partial X_{1}} \exp \left[i\left(k^{\prime} X_{0}-\omega_{0} T_{0}\right)-k^{\prime} y\right] \\
& -E_{0}^{(2)} \frac{\tilde{\varepsilon}^{(2)}-\tilde{\varepsilon}^{(1)}}{\tilde{\varepsilon}^{(2)}+\tilde{\varepsilon}^{(1)}}\left(\Omega_{E+}+k^{\prime}\right) D^{2} \exp \left[2 i\left(k^{\prime} X_{0}-\omega_{0} T_{0}\right)-2 k^{\prime} y\right]+\text { c.c., } \\
\psi_{2}^{(1)}= & -i E_{0}^{(1)} \frac{\tilde{\varepsilon}^{(2)}-\tilde{\varepsilon}^{(1)}}{\tilde{\varepsilon}^{(2)}+\tilde{\varepsilon}^{(1)}} y \frac{\partial D}{\partial X_{1}} \exp \left[i\left(k^{\prime} X_{0}-\omega_{0} T_{0}\right)+k^{\prime} y\right] \\
& +E_{0}^{(1)} \frac{\tilde{\varepsilon}^{(2)}-\tilde{\varepsilon}^{(1)}}{\tilde{\varepsilon}^{(2)}+\tilde{\varepsilon}^{(1)}}\left(\Omega_{E+}-k^{\prime}\right) D^{2} \exp \left[2 i\left(k^{\prime} X_{0}-\omega_{0} T_{0}\right)+2 k^{\prime} y\right]+\text { c.c., } \\
\phi_{2}^{(2)}=- & \frac{1}{k^{\prime}}\left[\frac{\partial D}{\partial T_{1}}+\frac{\partial D}{\partial X_{1}}\left(-V^{(2)} k^{\prime} y+\frac{\omega_{0}}{k^{\prime}}\left(1+k^{\prime} y\right)\right)\right] \exp \left[i\left(k^{\prime} X_{0}-\omega_{0} T_{0}\right)-k^{\prime} y\right] \\
- & i\left(V^{(2)}-\frac{\omega_{0}}{k^{\prime}}\right)\left(\Omega_{E+}+k^{\prime}\right) D^{2} \exp \left[2 i\left(k^{\prime} X_{0}-\omega_{0} T_{0}\right)-2 k^{\prime} y\right]+\text { c.c.., } \\
\phi_{2}^{(1)}= & \frac{1}{k^{\prime}}\left[\frac{\partial D}{\partial T_{1}}+\frac{\partial D}{\partial X_{1}}\left(V^{(1)} k^{\prime} y+\frac{\omega_{0}}{k^{\prime}}\left(1-k^{\prime} y\right)\right)\right] \exp \left[i\left(k^{\prime} X_{0}-\omega_{0} T_{0}\right)+k^{\prime} y\right] \\
& +i D^{2}\left(V^{(1)}-\frac{\omega_{0}}{k^{\prime}}\right)\left(\Omega_{k+}-k^{\prime}\right) \exp \left[2 i\left(k^{\prime} X_{0}-\omega_{0} T_{0}\right)+2 k^{\prime} y\right]+\text { c.c., }(A .1)
\end{aligned}
$$


where $\Omega_{l i+}$ is given by Eq. (3.4). The solutions of the second-order problem yield the solvability condition given by Eq. (3.1).

For order $\varepsilon^{3}$.

$$
\begin{aligned}
& \frac{\partial^{2} \psi_{3}^{(2)(1)}}{\partial X_{0}^{2}}+\frac{\partial^{2} \psi_{3}^{(2)(1)}}{\partial y^{2}}=-2 \frac{\partial^{2} \psi_{2}^{(2)(1)}}{\partial X_{0} \partial X_{1}}-2 \frac{\partial^{2} \psi_{1}^{(2)(1)}}{\partial X_{0} \partial X_{2}}-\frac{\partial^{2} \psi_{1}^{(2)(1)}}{\partial X_{1}^{2}} \\
& \frac{\partial^{2} \phi_{3}^{(2)(1)}}{\partial X_{0}^{2}}+\frac{\partial^{2} \phi_{3}^{(2)(1)}}{\partial y^{2}}=-2 \frac{\partial^{2} \phi_{2}^{(2)(1)}}{\partial X_{0} \partial X_{1}}-2 \frac{\partial^{2} \phi_{1}^{(2)(1)}}{\partial X_{0} \partial X_{2}}-\frac{\partial^{2} \phi_{1}^{(2)(1)}}{\partial X_{1}^{2}}, \\
& \llbracket \frac{\partial \psi_{3}}{\partial X_{0}} \rrbracket-\frac{\partial \eta_{3}}{\partial X_{0}} \llbracket E_{0} \rrbracket=\frac{\partial \eta_{2}}{\partial X_{1}} \llbracket E_{0} \rrbracket+\frac{\partial \eta_{1}}{\partial X_{2}} \llbracket E_{0} \rrbracket-\eta_{1} \frac{\partial \eta_{1}}{\partial X_{0}} \llbracket \frac{\partial^{2} \psi_{1}}{\partial \dot{y}^{2}} \rrbracket \\
& -\frac{\partial \eta_{1}}{\partial X_{0}} \llbracket \frac{\partial \psi_{2}}{\partial y} \rrbracket-\frac{\partial \eta_{2}}{\partial X_{0}} \llbracket \frac{\partial \psi_{1}}{\partial y} \rrbracket-\frac{\partial \eta_{1}}{\partial X_{1}} \llbracket \frac{\partial \psi_{1}}{\partial y} \rrbracket \\
& -\eta_{2} \llbracket \frac{\partial^{2} \psi_{1}}{\partial y \partial X_{0}} \rrbracket-\frac{1}{2} \eta_{1}^{2} \llbracket \frac{\partial^{3} \psi_{1}}{\partial y^{2} \partial X_{0}} \rrbracket-\eta_{1} \llbracket \frac{\partial^{2} \psi_{2}}{\partial y \partial X_{0}} \rrbracket \\
& -\eta_{1} \llbracket \frac{\partial^{2} \psi_{1}}{\partial y \partial X_{1}} \rrbracket-\llbracket \frac{\partial \psi_{2}}{\partial X_{1}} \rrbracket-\llbracket \frac{\partial \psi_{1}}{\partial X_{2}} \rrbracket, \quad \text { at } y=0, \\
& \left.\llbracket \tilde{\varepsilon} \frac{\partial \psi_{3}}{\partial y} \rrbracket=\eta_{1} \frac{\partial \eta_{1}}{\partial X_{0}} \llbracket \tilde{\varepsilon} \frac{\partial^{2} \psi_{1}}{\partial y \partial X_{0}} \rrbracket+\frac{\partial \eta_{1}}{\partial X_{0}} \llbracket \tilde{\varepsilon} \frac{\partial \psi_{2}}{\partial X_{0}}\right]+\frac{\partial \eta_{1}}{\partial X_{0}} \llbracket \tilde{\varepsilon} \frac{\partial \psi_{1}}{\partial X_{1}} \rrbracket \\
& +\left(\frac{\partial \eta_{2}}{\partial X_{0}}+\frac{\partial \eta_{1}}{\partial X_{1}}\right) \llbracket\left[\tilde{\varepsilon} \frac{\partial \psi_{1}}{\partial X_{0}}\right]-\eta_{2}\left[\tilde{\varepsilon} \frac{\partial^{2} \psi_{1}}{\partial y^{2}}\right] \\
& -\frac{1}{2} \eta_{1}^{2} \llbracket \tilde{\varepsilon} \frac{\partial^{3} \psi_{1}}{\partial y^{3}} \rrbracket-\eta_{1} \llbracket \tilde{\varepsilon} \frac{\partial^{2} \psi_{2}}{\partial y^{2}} \rrbracket, \quad \text { at } y=0 \text {, } \\
& \frac{\partial \eta_{3}}{\partial T_{0}}-\frac{\partial \phi_{3}^{(2)(1)}}{\partial y}+V^{(2)(1)} \frac{\partial \eta_{3}}{\partial X_{0}}=-\frac{\partial \eta_{1}}{\partial T_{2}}-\frac{\partial \eta_{2}}{\partial T_{1}}+\eta_{1} \frac{\partial^{2} \phi_{2}^{(2)(1)}}{\partial y^{2}} \\
& +\eta_{2} \frac{\partial^{2} \phi_{1}^{(2)(1)}}{\partial y^{2}}+\frac{1}{2} \eta_{1}^{2} \frac{\partial^{3} \phi_{1}^{(2)(1)}}{\partial y^{3}}-\frac{\partial \phi_{1}^{(2)(1)}}{\partial X_{0}} \frac{\partial \eta_{2}}{\partial X_{0}}-\frac{\partial \eta_{1}}{\partial X_{0}} \frac{\partial \phi_{2}^{(2)(1)}}{\partial X_{0}} \\
& -\frac{\partial \phi_{1}^{(2)(1)}}{\partial X_{1}} \frac{\partial \eta_{1}}{\partial X_{0}}-\frac{\partial \eta_{1}}{\partial X_{1}} \frac{\partial \phi_{1}^{(2)(1)}}{\partial X_{0}}-\eta_{1} \frac{\partial \eta_{1}}{\partial X_{0}} \frac{\partial^{2} \phi_{1}^{(2)(1)}}{\partial X_{0} \partial y} \\
& -V^{(2)(1)} \frac{\partial \eta_{2}}{\partial X_{1}}-V^{(2)(1)} \frac{\partial \eta_{1}}{\partial X_{2}}, \quad \text { at } y=0 \text {, }
\end{aligned}
$$




$$
\begin{aligned}
& \frac{\partial \phi_{3}^{(1)}}{\partial T_{0}}-\rho \frac{\partial \phi_{3}^{(2)}}{\partial T_{0}}+(1-\rho) \eta_{3}-\frac{\partial^{2} \eta_{3}}{\partial X_{0}^{2}}+V^{(1)} \frac{\partial \phi_{3}^{(1)}}{\partial X_{0}}-\rho V^{(2)} \frac{\partial \phi_{3}^{(2)}}{\partial X_{0}}+\llbracket \tilde{\varepsilon} E_{0} \frac{\partial \psi_{3}}{\partial y} \rrbracket \\
& =-\frac{\partial \phi_{1}^{(1)}}{\partial T_{2}}-\frac{\partial \phi_{2}^{(1)}}{\partial T_{1}}+\rho \frac{\partial \phi_{1}^{(2)}}{\partial T_{2}}+\rho \frac{\partial \phi_{2}^{(2)}}{\partial T_{1}} \\
& +2 \frac{\partial^{2} \eta_{1}}{\partial X_{0} \partial X_{2}}+2 \frac{\partial^{2} \eta_{2}}{\partial X_{0} \partial X_{1}}+\frac{\partial^{2} \eta_{1}}{\partial X_{1}^{2}}-\frac{\partial \phi_{1}^{(1)}}{\partial X_{0}} \frac{\partial \phi_{2}^{(1)}}{\partial X_{0}}-\frac{\partial \phi_{1}^{(1)}}{\partial X_{0}} \frac{\partial \phi_{1}^{(1)}}{\partial X_{1}} \\
& -\eta_{1} \frac{\partial \phi_{1}^{(1)}}{\partial X_{0}} \frac{\partial^{2} \phi_{1}^{(1)}}{\partial y \partial X_{0}}+\rho \frac{\partial \phi_{1}^{(2)}}{\partial X_{0}} \frac{\partial \phi_{2}^{(2)}}{\partial X_{0}}+\rho \frac{\partial \phi_{1}^{(2)}}{\partial X_{0}} \frac{\partial \phi_{1}^{(2)}}{\partial X_{1}}+\rho \eta_{1} \frac{\partial \phi_{1}^{(2)}}{\partial X_{0}} \frac{\partial^{2} \phi_{1}^{(2)}}{\partial y \partial X_{0}} \\
& -\frac{\partial \phi_{1}^{(1)}}{\partial y} \frac{\partial \phi_{2}^{(1)}}{\partial y}-\eta_{1} \frac{\partial \phi_{1}^{(1)}}{\partial y} \frac{\partial^{2} \phi_{1}^{(1)}}{\partial y^{2}}+\rho \frac{\partial \phi_{1}^{(2)}}{\partial y} \frac{\partial \phi_{2}^{(2)}}{\partial y}+\rho \eta_{1} \frac{\partial \phi_{1}^{(2)}}{\partial y} \frac{\partial^{2} \phi_{1}^{(2)}}{\partial y^{2}} \\
& -\eta_{2} \frac{\partial^{2} \phi_{1}^{(1)}}{\partial T_{0} \partial y}-\eta_{1} \frac{\partial^{2} \phi_{2}^{(1)}}{\partial T_{0} \partial y}-\frac{1}{2} \eta_{1}^{2} \frac{\partial^{3} \phi_{1}^{(1)}}{\partial T_{0} \partial y^{2}}+\rho \eta_{2} \frac{\partial^{2} \phi_{1}^{(2)}}{\partial T_{0} \partial y} \\
& +\rho \eta_{1} \frac{\partial^{2} \phi_{2}^{(2)}}{\partial T_{0} \partial y}+\frac{1}{2} \rho \eta_{1}^{2} \frac{\partial^{3} \phi_{1}^{(2)}}{\partial T_{0} \partial y^{2}}-\eta_{1} \frac{\partial^{2} \phi_{1}^{(1)}}{\partial T_{1} \partial y}+\rho \eta_{1} \frac{\partial^{2} \phi_{1}^{(2)}}{\partial T_{1} \partial y}-\frac{3}{2} \frac{\partial^{2} \eta_{1}}{\partial X_{0}^{2}}\left(\frac{\partial \eta_{1}}{\partial X_{0}}\right)^{2} \\
& -V^{(1)}\left[\eta_{2} \frac{\partial^{2} \phi_{1}^{(1)}}{\partial y \partial X_{0}}+\frac{1}{2} \eta_{1}^{2} \frac{\partial^{3} \phi_{1}^{(1)}}{\partial y^{2} \partial X_{0}}+\eta_{1} \frac{\partial^{2} \phi_{1}^{(1)}}{\partial y \partial X_{0}}+\eta_{1} \frac{\partial^{2} \phi_{1}^{(1)}}{\partial y \partial X_{1}}+\frac{\partial \phi_{2}^{(1)}}{\partial X_{1}}+\frac{\partial \phi_{1}^{(1)}}{\partial X_{2}}\right] \\
& +\rho V^{(2)}\left[\eta_{2} \frac{\partial^{2} \phi_{1}^{(2)}}{\partial y \partial X_{0}}+\frac{1}{2} \eta_{1}^{2} \frac{\partial^{3} \phi_{1}^{(2)}}{\partial y^{2} X_{0}}+\eta_{1} \frac{\partial^{2} \phi_{2}^{(2)}}{\partial y \partial X_{0}}+\eta_{1} \frac{\partial^{2} \phi_{1}^{(2)}}{\partial y \partial X_{1}}+\frac{\partial \phi_{2}^{(2)}}{\partial X_{1}}+\frac{\partial \phi_{1}^{(2)}}{\partial X_{2}}\right] \\
& -\eta_{1} \llbracket \tilde{\varepsilon} \frac{\partial \psi_{1}}{\partial X_{0}} \frac{\partial^{2} \psi_{1}}{\partial X_{0} \partial y} \rrbracket-\llbracket \tilde{\varepsilon} \frac{\partial \psi_{1}}{\partial X_{0}} \frac{\partial \psi_{2}}{\partial X_{0}} \rrbracket-\llbracket \tilde{\varepsilon} \frac{\partial \psi_{1}}{\partial X_{0}} \frac{\partial \psi_{1}}{\partial X_{1}} \rrbracket-\eta_{2} \llbracket \tilde{\varepsilon} E_{0} \frac{\partial^{2} \psi_{1}}{\partial y^{2}} \rrbracket \\
& -\frac{1}{2} \eta_{1}^{2} \llbracket \tilde{\varepsilon} E_{0} \frac{\partial^{3} \psi_{1}}{\partial y^{3}} \rrbracket-\eta_{1} \llbracket \tilde{\varepsilon} E_{0} \frac{\partial^{2} \psi_{2}}{\partial y^{2}} \rrbracket+\eta_{1} \llbracket \tilde{\varepsilon} \frac{\partial \psi_{1}}{\partial y} \frac{\partial^{2} \psi_{1}}{\partial y^{2}} \rrbracket \\
& +\llbracket \tilde{\varepsilon} \frac{\partial \psi_{1}}{\partial y} \frac{\partial \psi_{2}}{\partial y} \rrbracket+2 \eta_{1} \frac{\partial \eta_{1}}{\partial X_{0}} \llbracket \tilde{\varepsilon} E_{0} \frac{\partial^{2} \psi_{1}}{\partial X_{0} \partial y} \rrbracket+2 \frac{\partial \eta_{1}}{\partial X_{0}} \llbracket \tilde{\varepsilon} E_{0} \frac{\partial \psi_{2}}{\partial X_{0}} \rrbracket \\
& +2 \frac{\partial \eta_{1}}{\partial X_{0}} \llbracket \tilde{\varepsilon} E_{0} \frac{\partial \psi_{1}}{\partial X_{1}} \rrbracket+2 \frac{\partial \eta_{2}}{\partial X_{0}} \llbracket \tilde{\varepsilon} E_{0} \frac{\partial \psi_{1}}{\partial X_{0}} \rrbracket+2 \frac{\partial \eta_{1}}{\partial X_{1}} \llbracket \tilde{\varepsilon} E_{0} \frac{\partial \psi_{1}}{\partial X_{0}} \rrbracket \\
& -2 \cdot \frac{\partial \eta_{1}}{\partial X_{0}} \llbracket \tilde{\varepsilon} \frac{\partial \psi_{1}}{\partial X_{0}} \frac{\partial \psi_{1}}{\partial y} \rrbracket-\left[2 \frac{\partial \eta_{1}}{\partial X_{0}} \frac{\partial \eta_{2}}{\partial X_{0}}+2 \frac{\partial \eta_{1}}{\partial X_{0}} \frac{\partial \eta_{1}}{\partial X_{1}}\right] \llbracket \tilde{\varepsilon} E_{0}^{2} \rrbracket \\
& +2\left(\frac{\partial \eta_{1}}{\partial X_{0}}\right)^{2}\left[\tilde{\varepsilon} E_{0} \frac{\partial \psi_{1}}{\partial y}\right], \quad \text { at } y=0 \text {. }
\end{aligned}
$$

The solutions of the above set of equations yield the solvability condition given by Eq. (3.2). 
Acknowledgments. The author wishes to express his cordial thanks to Professor D. G. Crighton for his critical reading of the manuscript. He is also grateful to Dr. A. A. Mohamed for various helpful discussions.

\section{REFERENCES}

[1] S. Chandrasekhar, Hydrodlnamic and hydromagnetic stability, Oxford University Press (1961)

[2] I. D. Chang and P. E. Russell, Stability of a liquid adjacent to a high-speed gas stream, Phys. Fluids 8, 1018 (1965)

[3] A. Davey, J. Fluid Mech. 53, 769 (1972)

[4] A. Davey and K. Stewartson, Proc. R. Soc. London A338, 101 (1974)

[5] P. (i. Drazin. J. Fluid Mech. 42, 321 (1970)

[6] E. F. El Shehawey and A. A. Mohamed, Nonlinear Kelvin-Helmholtz instability effect of a tangential electric field. to appear

[7] R. L. (jater and M. R. L'Ecuyer, A fundamental investigation of the phenomena that characterize liquid film cooling. Purdue University Rep. No. Tm. 69-1 (1969)

[8] H. (jold, J. H. Otis and R. E. Schlier, Surface liquid film characteristics: an experimental study, A.I.A.A. Paper No. $71-623$ (1971)

[9] H. Hasimoto and H. Ono, J. Phys. Soc. Japan 33, 805 (1972)

[10] R. Kant and S. K. Malik, Astrophys. space Sc. 86, 345 (1982)

[11] V. I. Karpman. Nonlinear waves in dispersive media, Pergamon Press (1975)

[12] W. Thomson, Lord Kelvin, The influence of wind on waves in water supposed frictionless, Phil. Mag. (4) 42. 368. Also in Mathematical and physical papers, vol. 4, p. 76, Cambridge University Press (1871)

[1.3] M. J. Lighthill, J. Inst. Math. Appl. 1, 269 (1965)

[14] S. A. Maslowe and R. E. Kelley, Int. J. Nonlinear Mech. 5, 427 (1970)

[15] J. R. Melcher, Field coupled surface waves, MIT Press, (1963)

[16] J. W. Miles, Envelope soliton problem, Siam. J. Appl. Math. 41, no. 2, 227-230 (1981)

[17] A. A. Mohamed and E. F. El Shehawey, Nonlinear electrohydrodynamic Rayleigh-Taylor instability. Part I, $A$ perpendicular field in the absence of surface charges, J. Fluid Mech. 129, 473-494 (1983)

[18] A. A. Mohamed and E. F. El Shehawey, Nonlinear electrohydrodynamic Rayleigh-Taylor instability'. II: A perpendicular field producing surface charge, Phys. Fluids 26, 1724 (1983)

[19] A. A. Mohamed and E. F. El Shehawey, Nonlinear electrohydrodynamic Rayleigh-Taylor instability. III Effect of a tangential field, AJSE 9, no. 4, 345-360 (1984)

[20] A. A. Mohamed and E. F. El Shehawey, Nonlinear Kelvin-Helmholtz instability, effect of a normal electric field. to appear (in Phys. Fluids)

[21] A. H. Nayfeh and W. S. Saric, Nonlinear Kelvin-Helmholtz instability, J. Fluid Mech. 46, 209 (1971)

[22] A. H. Nayfeh and W. S. Saric, J. Fluid Mech. 55, 311 (1972)

[23] A. H. Nayfeh and W. S. Saric, J. Fluid Mech. 58, 39 (1973a)

[24] A. H. Nayfeh, Perturbation methods, Wiley Interscience (1973b)

[25] A. H. Nayfeh, Trans. A.S.M.E.E. J. Appl. Mech. 43, 584 (1976)

[26] W. S. Saric and B. W. Marshall, An experimental investigation of the stability of a thin liquid layer adjacent to a supersonic stream, A.I.A.A.J. 9, 1546 (1971)

[27] A. C. Scott, F. Y. F. Chu and D. W. McLaughlin, Proc. IEEE 61, 1443 (1973)

[28] C. V. Smith and J. R. Melcher, Phys. Fluids 10, 2315 (1967)

[29] W. Strauss. Nonlinear problems on theoretical physics (ed. A. F. Rañada), Lecture Notes in Physics, vol. $98, p$. 127. Springer (1979)

[30] (3. I. Taylor, Proc. R. Soc. London A313, 453 (1969)

[31] S. A. Thorpe, J. Fluid Mech. 32, 693 (1968)

[32] S. A. Thorpe, J. Fluid Mech. 39, 25 (1969)

[33] S. A. Weissman, Phil. Trans. 290A, 639 (1979)

[34] (j. B. Whitham, Linear and nonlinear waves, John Wiley, New York (1974)

[35] H. H. Woodson and J. R. Melcher, Electromechanical dynamics, Part II, John Wiley (1968)

[36] V. E. Zakharov and A. B. Shabat, Soviet Phys. JETP 34. 62 (1972) 